\title{
Video Post Processing Method For On Board Vehicle Camera with Integrated Eye Tracker
}

\author{
Juraj PANČÍK**, Pavel MAXERA, Robert KLEDUS, Michal BELÁK, Martin BILÍK \\ \{juraj.pancik@gmail.com, robert.kledus@usi.vutbr.cz, \\ pavel.maxera@usi.vutbr.cz, michal.belak@usi.vutbr.cz,martin.bilik@usi.vutbr.cz\}
}

Institute of Forensic Engineering, Brno University of Technology, Purkyňova,4641/118, Brno, 61200, Czech Republic, phone of *: +421 908963289

\begin{abstract}
This article describes how to process Eye Tracker System (ETS) data from recorded videos. The research task consisted in confirming the literature fact that the eyes of the moving person (in our case the driver in the car) inadvertently concentrate their position of the eye's sharp vision center (ESVC) on the place on the scene where the center of the optical flow is located. ETS video records were obtained during experiments in a real vehicle test environment. As part of the post-processing of ETS videos, we determined the numerical difference between the sharp eye viewing position center and the center of the optical flow center (FOE, focus of expansion) for each recorded image. In video post processing, the vibration of the driver's head in moving car were corrected at it was based on recorded acceleration data. The correcting of acceleration data from the ETS had significantly improved the results of the difference assessment of both centers - ESVC and FOE. A program framework was created in the MATLAB computing environment and it is ready for future use. This work can be useful as contribution in development of driver monitoring systems and fatigue detection software development and road safety improvement. Lack of concentration in a driver due to fatigue is a major cause of road accidents. This approach (the measurement of distance between ESVC and FOE positions) can be used in role input data generator to develop video processing and artificial intelligence based system to automatically detect driver fatigue and warn the driver, in order to prevent accidents.
\end{abstract}

Keywords: eye tracker system, optical flow, driver monitoring systems, fatigue detect software

\section{Introduction}

In this article is describing an image processing method for videos recorded by driver's eye tracker system (in next as ETS, Eye Tracker System).Video stream and data were obtained during experiments with vehicle. The ETS consists from "eyeglasses" which are carried by driver during experiment and from "system unit". ETS's eyeglasses are occupied with source of infrared (IR) light (two IR LED, one for each eye), two CCD/CMOS cameras and image processing system for determination of position eye's sharp vision with 1 grade accuracy. ETS also contains one "forward looking camera" (FVC). This FVC imaging camera is directed along the driver's sight. ETS system unit captured video streams for all three cameras, processes them and created output mixed video stream. This mixed video stream contains output video stream from FLC and added special symbols represented position of eye's sharp vision. ETS system unit captured another physical values recorded during car's movement. 
ETS output data therefore have character of video records from FLC with mixed information about eye's sharp vision center position (ESVC) represented by one circle. Recorded videos from ETS are influenced by strong vibrations in dependence of road quality and this makes things worse. By us designed and verified method for suppression of vibrations in recorded videos (ETS video processing) can help in quantitative and qualitative analysis of driver's eyes movement during driving.

\subsection{Driver Monitoring System}

Driver drowsiness detection is a car safety technology which helps prevent accidents caused by the driver getting drowsy. Various studies have suggested that around $20 \%$ of all road accidents are fatigue-related, up to $50 \%$ on certain roads. Some of the current systems learn driver patterns and can detect when a driver is becoming drowsy [1]. Modern systems consists DMS (Driver Monitoring System) [2] and software for detection fatigue (FDS, Fatigue Detection Software) [3]. From market point of view according to study LC Technologies in 2019 year will be sold about 6.7 million of DMS based on embedded in cars eye tracking technology [4]. As an actual example for luxury cars should be served the solution from HARMAN [5]. The survey of ETS technology is in [6]. The study of movement of driver's eyes with eye tracker technologies is long-term study field. The survey of results and of literature can be reach in [7] for example.

\subsection{Optical flow, focus of expansion and human eye movement}

As we mentioned in previous, the ETS forward looking camera generates a set of images (video sequence, video stream) and this set of frames can be understand as dynamic image. Properties of dynamic image can be very well described by using the optical flow concept and its parameters. The survey of sources is in [8]. Parameters of optical flow can be represented by set of vectors of optical flow. The relationships between eye's movement and scene's optical flow have been studied [9] [10] [11].

During walking or driving, the optical flow field an observer expands from a singular point, called the focus of expansion (FOE). Driver's eyes movement have been studied before [7] [12]. All visual motion is directed radial away from the focus of expansion, which lies in the direction of the heading of the car. In this situation, one may ask whether the driver can use the location of the focus of expansion to estimate his or her heading. Several studies have shown that humans can accurately locate the focus of expansion in the optic flow and suggested that this capability may contribute to driving behavior. In next we mention of results from one significant study from Fukuchi and others [13]. According this study the FOE in the field of view attracts significant amount of task-independent attention. Fukuchi and others [13] state that observers' overt attention (i.e., eye movements) was strongly drawn to the FOE, both when viewing natural scenes as well as in visual search. The effects were stronger and remained longer than any other cues tested (intensity, color, flicker, perspective, contractive motion). Authors compared the performance of the saliency model of visual attention (published model) with or without the FOE component. By incorporating the FOE component, the model predicted the human eye movements significantly better. 


\section{Problem definition}

Our problem we can describe as confirmation of experimental observation which was very well described in literature. This observation (and our hypothesis) states that eyes of a movement person (in our case a driver in movement car) naturally concentrate its eye's sharp vision centre position (therefore somewhere on road) where is localized the center of optical flow (focus of expansion, FOE) [9] [10]. This naturally case of driver's sight is not valid for cases where driver active focuses his sight at objects in his field of view. The center of optical flow in field of view of objects in movement state (person, car or locomotive) can be determined by methods of image processing of video stream produced by object's camera. This object's camera is directed along direction of object movement. The object's camera in the ETS is represented by forward looking camera (FLC). The FLC is placed on driver's head as a part of ETS "eyeglasses".

The position of eye's sharp vision centre in by driver observed scene is determined by special subsystem of the ETS and this subsystem "mixed" calculated position of eye's sharp vision centre and video output from FLC. The Figure 1 shows one frame from our ETS output video sequence during real experiments. For purpose of confirmation above mentioned hypothesis we had a set of experimental data. These data consists from video records from ETS [13] in real vehicle experiments carried out in our department [14] [15]. In this video records is visible the center of eye's sharp vision as circle with smaller radius (Figure 1).

When we saw of video records from ETS captured during real vehicle tests we pointed out a negative role of vibrations (vibrations of moving car and driver's head) to calculate of center of optical flow. Inherent frequency spectrum of vibrations of driver's head (ETC with FVC) and inherent frequency spectrum of vibrations of moving car are qualitative and quantitative different. Both types of vibrations have different amplitudes and different frequency spectrum. In the captured FVC frame sequences both vibrations can be accepted as differential (both direction vector and acceleration vector are different but mutually dependent each other). The exact mathematical model of vibration is not possible to build. Besides of video records from ETS there is a GPS data file as a part of recorded data from ETS. Together with GPS data also XYZ accelerations data are recorded. These data represent of car's vibration.

\section{Problem analysis}

\subsection{Inputs requirements}

For solving of our problem we have files with recorded "raw" video signal from ETS FLC without any mixed information (Figure 3). As next we have another video record from ETS FLS with mixed info about position of eye's sharp vision center (see Figure 1). This mixed information (besides video from scene) consists from two circles - inner circle represents position eye's sharp vision center (ESVC) and outer circle represents driver's field of view. Both concentric circles origin from video post processing system of both ETS eye's position track cameras based on IR LED light. Vibrations of movement vehicle can be represents also from point of view a static observer somewhere on the road. The vehicle is moving from one side to another, rotates about its axial axis and changes direction of movement.

Driver's eye naturally corrects a head's movement with inherent frequency spectrum about $1 \mathrm{~Hz}$; it worse filters head's movement with frequency spectrum above $1 \mathrm{~Hz}$. This eye's 
property has evolution background [10]. Eye's movement is connected with head's movement and it is influenced thanks to accelerations sensor in the brain [16]. According to our observation the video record from driver's ETS shows more kinds of behavior of eye's sharp vision center: spontaneous setting s or cognitive stimulated settings (traffic signs or walking pedestrian on the border of road, vehicle's dashboard). We also observed temporary limited concentration of driver's sight towards parts of scene without any significant mark - this fact is supported also by literature [12] [16].

\subsection{Outputs requirements}

We will use of image processing methods for processing of video records from ETS (non real time image processing). From recorded images we want to determine the position of eye's sharp vision from center of radius smaller circle (Figure 1) for each frame. From recorded "raw" videos (source is the forward looking camera mounted in ETS) we want to determine position of expansion point (center of optical flow vectors) (Figure 2). After determination of both positions in X-Y frame's axis we want for each frame calculate difference between them in vector's terms - length of vector (CCD sensor pixels unit) and vector angle (degree unit) (Figure 3). The output differential vector (length, angle) is created with center in position of eye's sharp vision center and the position of actual calculated expansion point represents the end point of vector. Zero azimuth (or zero angle) of vector is referenced to the actual value of gravity vector - this information is transferred from GPS unit and is updated with frame periodicity.

\subsection{Requirements for algorithms}

A. From ETS recorded video sequences calculate a position of eye's sharp vision center. To determine this position use position of center of radius of smaller circle which represent the sharp vision of driver (Figure 1)

B. From ETS recorded "raw" video sequences calculate optical flow vectors and determine the focus of expansion, FOE (center of optical flow vectors) (Figure 2)

C. During video post processing is necessary to correct influence of vibrations of moving car. For this purpose use recorded by GPS unit acceleration data in X, Y, Z axis with frame periodicity. Data from head's acceleration are not available, but in the future we expect add this option.

\section{Problem solving}

\subsection{System and hardware environment}

We carried out experiments with vehicle on real roads and hours of videos from ETS (both, raw from FLC and with eye tracking processing) was recorded together with data from GPS. As eye tracker system we use the ETS from View Point System [14]. Necessary calibration of ETS was performed before each trial in vehicle (night and day sessions). This calibration ensures correct and accuracy measurements of eye position with ETS embedded IR LED lights and cameras. The description of calibration procedure is beyond of scope this article. 
The overall system is described in Figure 3. System serves for calculation of position differences for eye's sharp vision center (ESVC) (carrying out in the ETS) and for calculated focus of expansion, FOE, as center of optical flow vectors for each recorded frame. Resulting graph represents in term of vectors these position differences in absolute length (sensor pixels as unit) and angle (or azimuth, degree as unit). Information about position difference between ESVC and FOE serves for representation of correction effectiveness of vehicle vibration during post processing process. If we have reasonable level of suppression of vehicle vibration we can track by this manner driver's eyes concentration on the road during driving. Lack of concentration in a driver due to fatigue is a major cause of road accidents. This approach measurement distance between ESVC and FOE can be used to develop a video-based system to automatically detect driver fatigue and warn the driver, in order to prevent accidents.

\subsection{Algorithms realization in MATLAB environment}

All algorithms we carried out in MATLAB environment (MATLAB ver.2017b, Image Processing Toolbox, Video Processing Toolbox). MATLAB has broad range of functions to manipulate with recorded videos. In order to determine position of ESVC we used position of center of radius of smaller (inner) circle which represent the sharp vision of driver (Figure 1). Circular Hough transforms can detect circles and their centers in images. At start in order to find circles and their centers we used the CircleFinder MATLAB application as circular Hough transform demo application. This application provides an interactive environment for changing parameters and options and it can be used to immediately visualize the effects of the calculation. In our program MATLAB function imFindCircles with by CircleFinder application found parameters was finally applied to determine ESVC.

Similar, standard MATLAB functions were utilized for calculation of optical flow and position of FOE (opticalFlow class and associated methods) for each frame in recorded videos.

In order to suppress vibrations effect we had to transform $\mathrm{X}, \mathrm{Y}, \mathrm{Z}$ acceleration data to more usable form for carry out a suppression image displacement. We applied the quaternion's theory and its application is described by Madgwick [18]. Generally the IMU (Inertial Measurement Unit) consists from gyroscopes and accelerometers enabling the tracking of rotational and translational movements from measured $\mathrm{X}, \mathrm{Y}, \mathrm{Z}$ acceleration data provided by GPS ETS unit in our case. For image transformations lead to suppression of effect vehicle vibrations the calculation of yaw rate, longitudinal and lateral acceleration from by GPS ETS unit measured X, Y, Z accelerations data we utilized a MATLAB library with name "Madgwick filter" associated also with source [18]. More detail description of these vibration suppression calculations is beyond of scope of this article.

\section{Experimental results and conclusions}

By exploration of experimental data with by us developed MATLAB framework we confirmed of experimental observations which were very well described in literature. We observed that driver's eyes during movement of car during significant part of time naturally concentrate the eye's sharp vision centre (ESVC) position near of center of optical flow (focus of expansion, FOE). Because there are two objective facts (automatic calculation of FOE and by driver's brain naturally alignment of position ESVC and FOE) we can utilize both facts to estimate objective level of driver's fatigue. There are few techniques to estimate driver's 
fatigue level which are described in literature and we assume that our approach (coincidence of FOE and ESVC positions) is new and should be study in details. We assume that suppression of vehicle's vibrations in the ETS data post processing is a actual topic. For example, the driver monitoring system for Lexus (Toyota) has appropriate use with only highways conditions when minimal vibrations are expected [5]. In order to calculate of FOE position in next research we recommend forward looking camera with $4 \mathrm{~K}$ resolution (ratio16:9, 4096 x 2304 pixels) and with raw video data outputs. In next research we recommend to use acceleration sensors integrated into ETS "glasseyes" to reach higher level of suppression of vibrations. The calculations of optical flow and FOE in real time are very hardware demanding and new methods to replace this approach should be find.This work can be useful as contribution in development of driver monitoring systems and fatigue detection software development and road safety improvement. Lack of concentration in a driver due to fatigue is a major cause of road accidents. This approach (the measurement of distance between ESVC and FOE positions) can be used in role input data generator to develop video processing and artificial intelligence based system to automatically detect driver fatigue and warn the driver, in order to prevent accidents.

\section{Acknowledgments}

This work was supported by Brno Technical University, Brno, Czech Republic, see projects [15][16] 


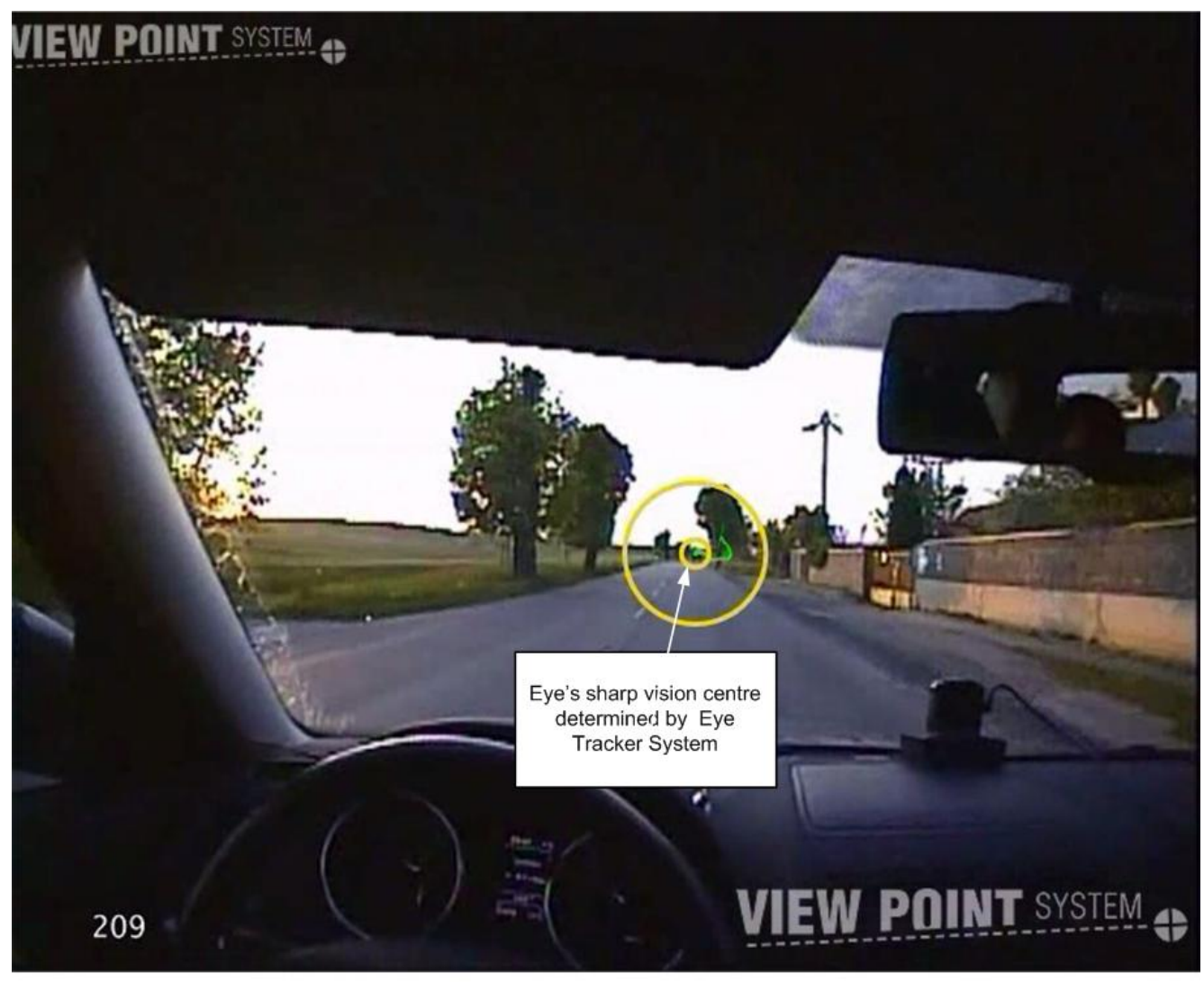

Figure 1. Video output from eye tracker system with mixed information about ESVC (Eye's Sharp Vision Center) 


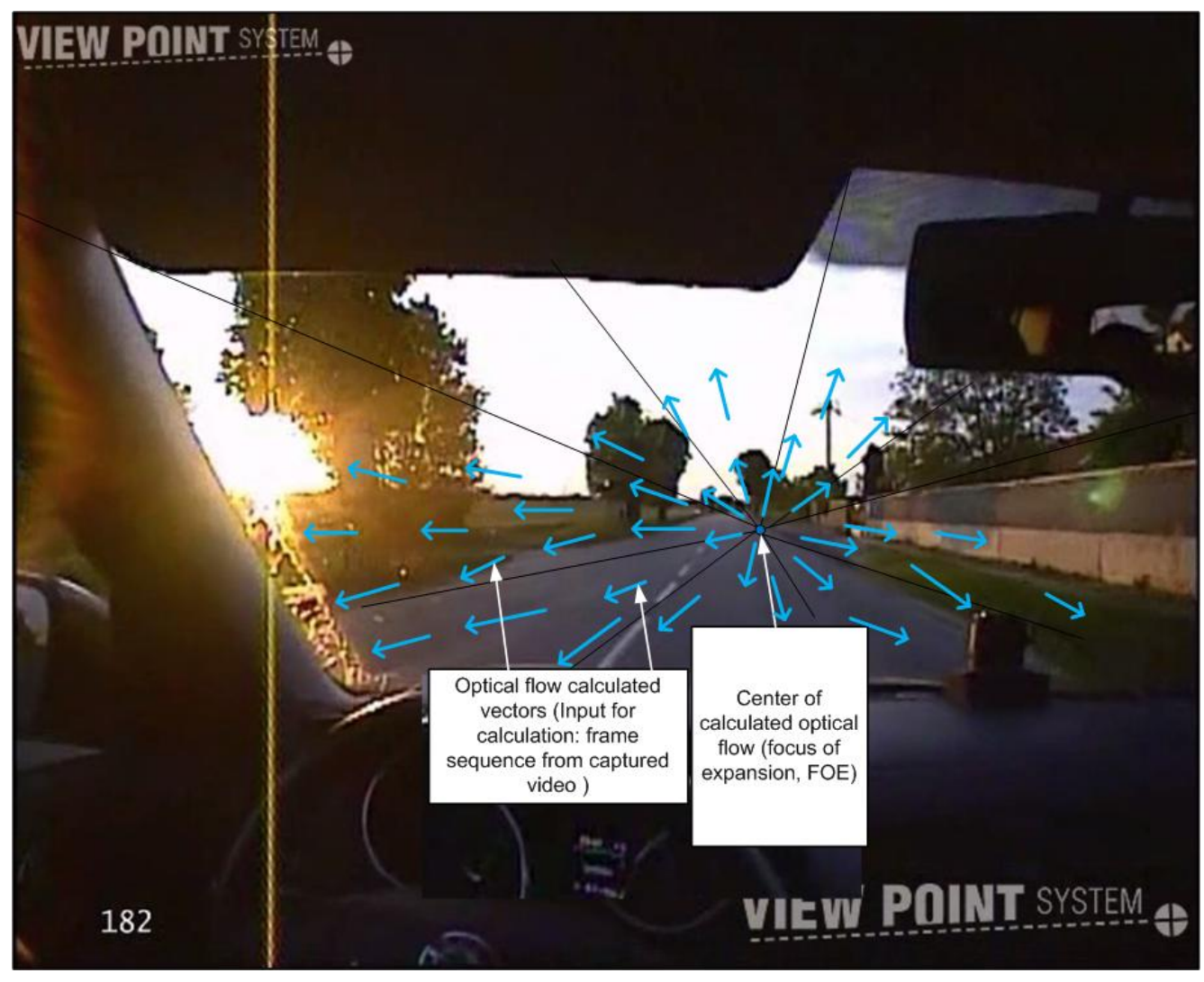

Figure 2. Optical flow vectors and calculated position of FOE (Focus of Expansion) 




Figure 3. System for ETS video post procesing 


\section{References}

[1] "Driver drowsiness detection," 2018. [Online]. Available: https://en.wikipedia.org/wiki/Driver_drowsiness_detection. [Accessed 13.1. 2018].

[2] "Driver Monitoring Systems," 2018. [Online]. Available:

https://en.wikipedia.org/wiki/Driver_Monitoring_System. [Accessed 13.8.2018].

[3] "Fatigue detection software," 2018. [Online]. [Accessed 13.7.2018].

[4]"Eye Tracking Technology is Making New Cars Safer," 2014. [Online]. Available:

http://www.eyegaze.com/eye-tracking-technology-is-making-new-cars-safer/. [Accessed 13.1.2018].

[5] K. RANJAN: "HARMAN introduces eye and pupils tracking for driver monitoring system,"

2016. [Online]. Available: http://telematicswire.net/harman-introduces-eye-and-pupils-tracking-fordriver-monitoring-system/. [Accessed 13.1.2018].

[6] AL-RAHAYFEH. A.: "Eye Tracking and Head Movement Detection: A State-of-Art Survey,"

IEEE Journal of Transactional engineering in Health and Medicine, 2013.

[7] J. GILLAND: "DRIVING, EYE-TRACKING AND VISUAL ENTROPY: EXPLORATION OF AGE AND TASK EFFECTS," Department of Psychology, The University of South Dakota, May 2008.

[8] "Optical flow," 2018. [Online]. Available: https://en.wikipedia.org/wiki/Optical_flow. [Accessed 13.7.2018].

[9] M. H.-P. LAPPE: "Optic Flow and Eye Movements," International Review of Neurobiology, vol.44, pp. 29-47, 2000.

[10] M. V. B. MOSSIO: "Traveled distances: New insights into the role of optic flow," Vision Research 48 , p. 289-303, 2008.

[11] W. H. WARREN: "Eye movements and optical flow," J.Opt.Soc.Am./Vol.7,No.1, January 1990, pp. 160-169, 1990.

[12] B. KAPITANIAK: "Application of Eye-Tracking in drivers Testing: A Reviewew o Research," International Journal of Occupational Medicine and Environmental Health, 28(6), pp. 941-954, 2015. [13] M. FUKUCHI, N. TSUCHYIA and C. KOCH: "The focus of expansion in optical flow fields acts as a strong cue for visual attention," Journal of Vision, no. 9(8), pp. 137, 137a, 2009, http://journalof vision.org/9/8/137/.

[14] VIEW POINT SYSTEM: "VPS Glasses, smart Unit, software," 2018. [Online]. Available: http://viewpointsystem.com/en/products/. [Accessed 14.1.2018].

[15] Institute of Forensic Engineering, Brno University of Technology, Brno, Czech Republic, Project ÚSI-J-16-3713 Analysis of driver's behaviour with eye tracking's method, Brno, Start: 01.01.2016, End: 31.12.2016.

[16] Institute of Forensic Engineering, Brno University of Technology, Brno, Czech Republic, Project TD020239 Technical assessment of traffic accidents at low visibility environment, Brno: VUT Brno, Start: 01.01.2014, End: 31.12.2015.

[17] A. WONG: Eye Movements Disorders, Oxford: Oxford University Press, 2007.

[18] S. MADGWICK: "An efficient orientation filter for inertial and inertial/magnetic sensor arrays" 2010. [Online]. Available: http://wiki.ros.org/imu_filter_madgwick. [Accessed 7/8/2018]. 